\title{
Comparison of Observed and Synthetic Spectra of AGB-Stars: A Progress Report
}

\author{
Stefan Uttenthaler ${ }^{1, *}$, Bernhard Aringer ${ }^{2}$, Michael Gorfer ${ }^{2}$, \\ Josef Hron ${ }^{2}$, Hans-Ulrich Käufl${ }^{1}$, and Thomas Lebzelter ${ }^{2}$ \\ ${ }^{1}$ ESO, Karl-Schwarzschildstr. 2, D-85748 Garching, Germany \\ email: suttenth@eso.org \\ ${ }^{2}$ Department of Astronomy, Türkenschanzstr. 17, A-1180 Vienna, Austria
}

\begin{abstract}
We have calculated a set of synthetic spectra for AGB-stars using the MARCScode (Gustafsson et al., 1975) and improved spectral synthesis. Here we investigate the spectral changes as a function of the stellar parameters and we show a comparison of observed high resolution UVES spectra of galactic disk and bulge AGB-stars with calculated synthetic spectra. Using our set of synthetic spectra, we try to determine the stellar parameters using only these and $(J-K)$ data (Schultheis 1998). To achieve this, we use a minimal-distance method and search for spectral features which can be used as indicators that are sensitive either only to a change in $T_{\text {eff }}$ or only to a change in metallicity $[M / H]$ (where $M$ are all metals from Li to $\mathrm{U})$. In a later stage of the project, we will focus on selected lines of s-process elements in an attempt to achieve a more accurate abundance determination leading to a better understanding of nucleosynthesis and dredge-up processes in these stars.
\end{abstract}

Keywords. Stars: AGB and post-AGB, stars: atmospheres, stars: abundances

\section{Introduction}

In previous works (Lebzelter \& Hron 2003, Uttenthaler et al. 2004) we studied the Technetium (Tc) content of samples of galactic disk and bulge stars, respectively, as a function of luminosity. As Tc is an unstable element $\left(\tau_{1 / 2} \simeq 2 \times 10^{5} \mathrm{yrs}\right)$ produced via the s-process, it is a reliable indicator of recent or ongoing third dredge-up (TDU). In first place, these works confirmed the estimated lower limit in luminosity for TDU to occur in thermally pulsating AGB-stars. At the same time, many luminous stars where found to be Tc-poor. From these findings motivation for a further investigation of the evolutionary status of these stars arose. For more details see the papers cited above.

\section{Constraining Stellar Parameters}

To do so, we conduct studies on the abundance of various s-process elements in our sample of bulge AGB-stars. For constraining current s-process nucleosynthesis models and for precise abundance determination it is of critical importance to derive accurate stellar parameters of the targets. For the determination of effective temperature $T_{\text {eff }}$, metallicity $[M / H]$ (where $M$ are all metals from Li to $\mathrm{U}$ ) and surface acceleration $\log (g)$ we use $(J-K)$ data (Schultheis 1998) and high resolution $(R \simeq 50000)$ UVES-spectra from the blue and red arm covering a large spectral range. The luminosity (distance) is rather well defined for the bulge stars.

The synthetic spectra we calculated are based on MARCS atmospheric models (Gustafsson et al. 1975), the spectral synthesis has been improved by additional opacity data 


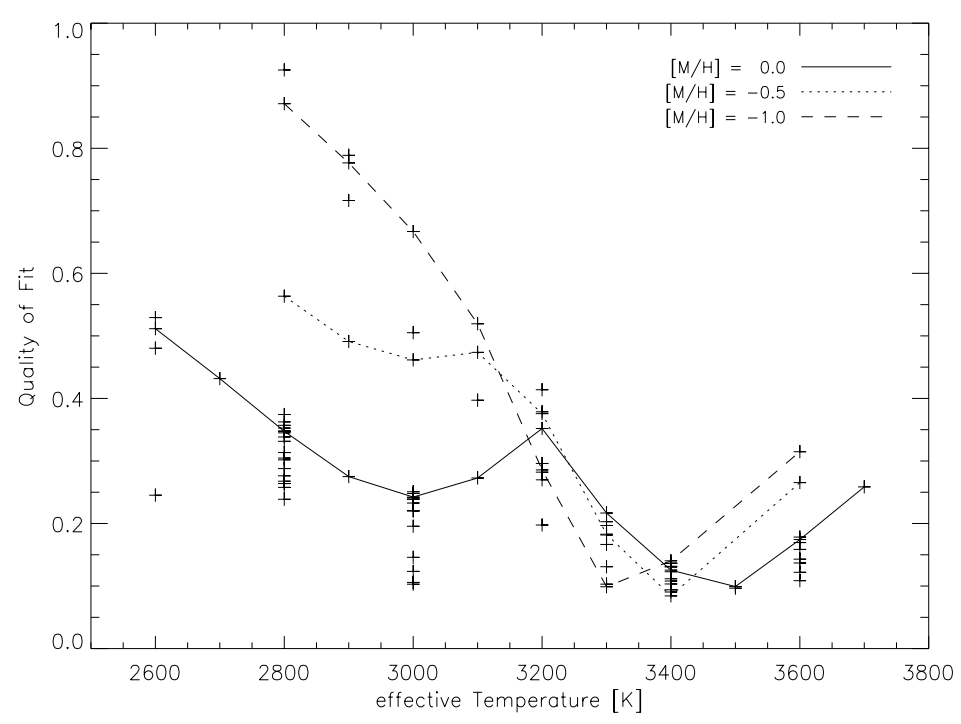

Figure 1. Quality of fit (i.e., distance to observed spectrum) for all 95 model spectra for a region including the $\mathrm{TiO}$ bandhead at $7056 \AA$. The observed spectrum used for this plot is that of a semi-regular bulge AGB-star. The lines connect data points of models with constant metallicity (and constant $\log (g)$ and luminosity) as indicated by the legend. The models with fairly low distance to the observed spectrum at $3000 \mathrm{~K}$ can be excluded as beeing the appropriate ones by comparison with other wavelength regions.

(including lines of Tc) and by including the second ionization stage of all elements, except $H$. The radiative transfer is done in spherical symmetry, consistent with the calculation of the atmospheric model.

For the comparison between observed and synthetic spectra we apply a minimal distance method to selected wavelength regions. Due to the absence of a reliable continuum, the flux of the synthetic spectrum $\left(F_{\lambda}\right)$ is normalized to the average of the observed flux. This procedure is followed for each of the atmospheric models using an IDL code. In principle, the synthetic spectrum yielding the lowest distance to the observed spectrum (i.e., lowest value for the "quality of fit", see Fig. 1) can be selected as the appropriate one, and the parameters of the underlying atmospheric model can be used as reasonable approximations to the real stellar parameters. As our analysis shows, for AGB-atmospheres this method leads to a degeneracy in the $\left(T_{e f f},[M / H]\right)$-space: A model of a certain $\left(T_{e f f},[M / H]\right)$-combination may fit the observed spectrum equally well as a cooler model with lower metallicity. In Fig. 1. this is illustrated for a 40 Åwide region containing the $\mathrm{TiO}$ bandhead at $7056 \AA$.

\section{Independent Indicators}

To overcome this degeneracy, we are searching for spectral features which are sensitive either only to a change in $T_{\text {eff }}$ or only to a change in $[M / H]$. For our search we defined a coarse grid of synthetic spectra and made plots of the ratio of the grid spectra and a reference spectrum, the latter being also a member of the coarse grid (all spectra are normalized to their mean flux). In these plots, strong spectral changes with changing model parameters are easily discernible. 
One line that could serve as an indicator for the metallicity is an iron line at $8468.45 \AA$. This line changes quite strongly as $[M / H]$ decreases. To be precise, the "continuum" goes up as the core flux of the line stays constant. Curiously, by reducing the metallicity, this line appears deeper! At the same time, the line almost doesn't change between $T_{\text {eff }}=2800 \mathrm{~K}$ and $T_{\text {eff }}=3200 \mathrm{~K}$. As with most of the indicators identified by now, they can only be used as such for a limited range in the stellar parameter space. For example, for $T_{\text {eff }}>3200 \mathrm{~K}$ the Fe line mentioned above changes with the temperature, too.

Line strength measurements of such indicators will finally be used to break the described degeneracies.

\section{References}

Gustafsson, B., et al. 1975, A\& A 42, 407

Lebzelter, T. \& Hron, J. 2003, A\&A 411, 533

Schultheis, M. 1998, Ph.D. thesis, University of Vienna, Austria

Uttenthaler, S., et al. 2004, Mem. S. A. It, 75, 590 


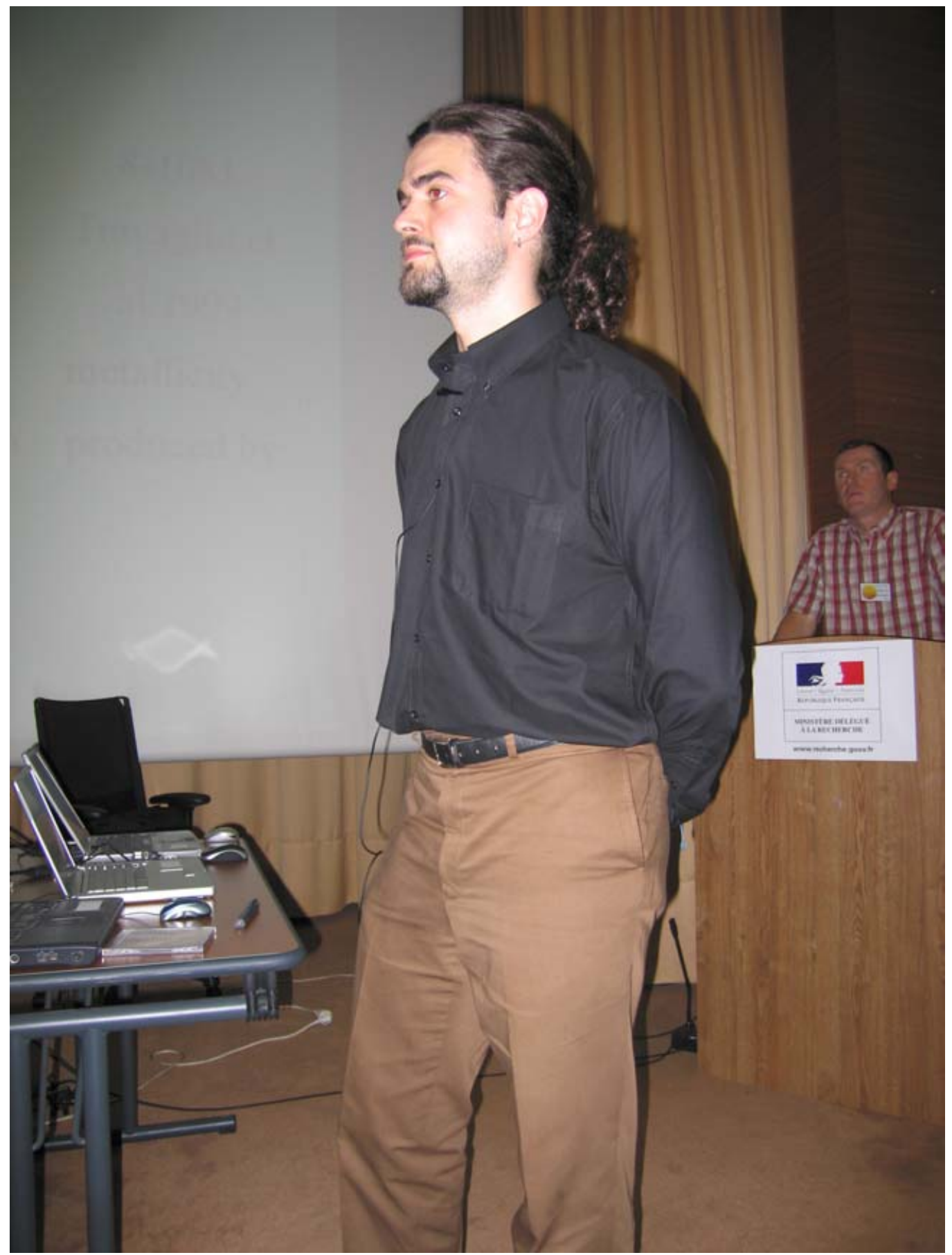

Gabriele Cescutti, talking about the chemical evolution of heavy elements in the Galaxy. 